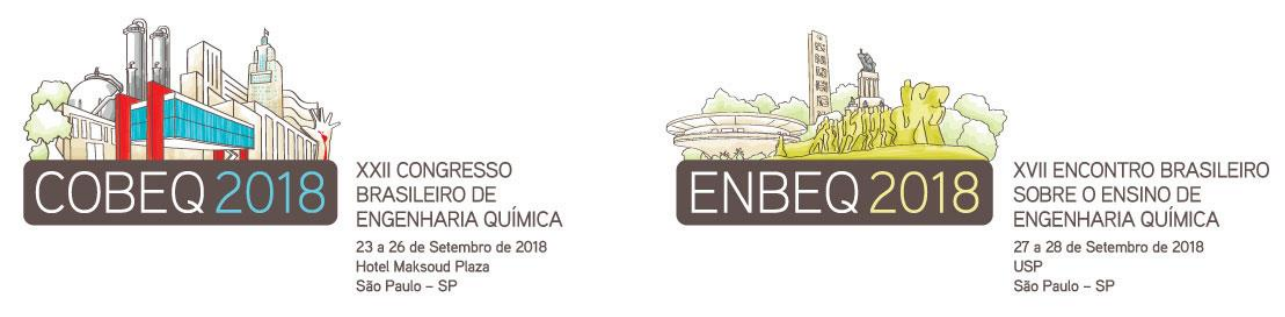

\title{
SÍNTESE DE NANOCOMPÓSITOS PMMA/NTCPM E CARACTERIZAÇÃO ELÉTRICA DE FILMES SEMICONDUTORES PRODUZIDOS VIA BLADE COATING
}

\author{
POLLI L ${ }^{1}$, BRESSANIN JM ${ }^{1}$, NETTO RA ${ }^{1}$, BARTOLI JR ${ }^{1}$, ALAFERDOV AV ${ }^{2}$, \\ HAMANAKA MHMO $^{3}$ e HAMANAKA VN ${ }^{4}$ \\ ${ }^{1}$ Universidade Estadual de Campinas, Faculdade de Engenharia Química, Departamento de \\ Engenharia de Materiais e de Bioprocessos \\ ${ }^{2}$ Universidade Estadual de Campinas, Centro de Componentes Semicondutores e Nanotecnologias \\ ${ }^{3}$ Centro de Tecnologia da Informação (CTI) Renato Archer \\ ${ }^{4}$ Universidade de São Paulo, Escola Politécnica, Depto.de Engenharia de Sistemas Eletrônicos
}

E-mail para contato: leo.polli@ hotmail.com

\begin{abstract}
RESUMO - Sintetizou-se o nanocompósito PMMA/NTCPM via polimerização in situ assistida por ultrassom visando a melhora na dispersão dos nanotubos na matriz polimérica. Foram produzidos filmes finos via casting e blade coating dos nanocompósitos. A incorporação dos nanotubos na matriz polimérica foi eficiente, evidenciado pelo caráter semicondutor dos filmes do nanocompósito sintetizado. As resistências de folha dos filmes finos semitransparentes obtidos por blade coating $(67$ a $93 \mathrm{k} \Omega / \mathrm{sq}$ ) são típicas de um material semicondutor, assim como para o filme produzido por casting $(3,82 \mathrm{k} \Omega / \mathrm{sq})$.
\end{abstract}

\section{INTRODUÇÃO}

Os nanotubos de carbono foram descobertos em 1991 por lijima, desde então vêm despertando grande interesse nos estudos que envolvem nanotecnologia, devido às suas excelentes propriedades mecânicas, elétricas e térmicas. Os nanotubos são especialmente interessantes quando utilizados como nanocargas em nanocompósitos poliméricos, pois suas excelentes propriedades podem ser incorporadas à matriz polimérica mesmo em teores relativamente baixos devido à elevada superfície específica das nanopartículas (Grossiord et al., 2006). O poli(metacrilato de metila) (PMMA) é um polímero da classe dos termoplásticos de uso comum e com elevada transparência e rigidez mecânica, de custo relativamente baixo. No presente trabalho objetiva-se incorporar as propriedades elétricas condutoras dos nanotubos de carbono à matriz polimérica de PMMA, que é um polímero não-condutor, de forma a obter um nanocompósito que una as características do PMMA com a capacidade condutora dos nanotubos. Filmes transparentes e condutores apresentam grande potencial de aplicação como eletrodos de dispositivos optoeletrônicos.

O maior desafio para incorporação das propriedades dos nanotubos de carbono de parede múltipla (NTCPM) à matriz polimérica é atingir um grau de dispersão uniforme das nanocargas na matriz. Todavia, isso é dificultado pelas interações entre nanotubos devido às forças de van der Waals que tendem a manter os nanotubos aglomerados (Ma et al., 2010). Com o objetivo de favorecer a dispersão dos nanotubos na matriz, diversos procedimentos 

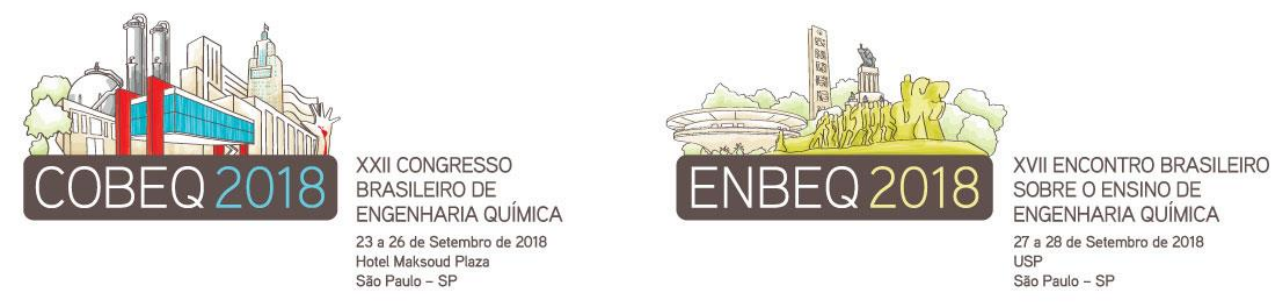

vêm sendo adotados no processo de síntese, dentre eles a polimerização in situ. Consiste na mistura das nanocargas com o monômero para posterior polimerização (Ma et al., 2010; Mittal et al., 2015). 0 uso de ultrassom tem sido usado para dispersar os aglomerados de NTCPM, e é também importante na etapa de iniciação da reação de polimerização. As reações sonoquímicas podem ocorrer devido ao fenômeno de cavitação das ondas de ultrassom, liberando alta energia e favorecendo a formação de radicais livres na reação (Suslick e Price, 1999). Nanocompósitos a base de PMMA/NTCPM com ótimas propriedades semicondutoras foram sintetizados neste grupo (Bressanin, 2017). A obtenção de filmes poliméricos condutores e transparentes é um grande desafio atual em ciência e tecnologia. Neste trabalho é apresentado o desenvolvimento de filmes finos de nanocompósitos semicondutores de PMMA/NTCPM produzidos por casting e blade coating.

\section{METODOLOGIA}

A síntese dos nanocompósitos foi realizada em escala laboratorial conforme procedimento adotado por Bressanin, 2017. Os NTCPM, fornecidos pelo Depto. de Física/UFMG, foram purificados em solução aquosa de $\mathrm{HCl} 5 \mathrm{~mol} / \mathrm{L}$ sob agitação, atingiu-se 98\% de pureza. O monômero de metil metacrilato, MMA (UNIGEL, 99,9\%), foi purificado para remoção da hidroquinona em coluna 306312 Sigma-Aldrich. As etapas da polimerização in situ foram: adição de $30 \mathrm{~mL}$ de clorofórmio ao béquer reacional e $0,1 \mathrm{~g}$ de NTCPM (1\% $\mathrm{m} / \mathrm{m}$ ), em seguida $10 \mathrm{~g}$ de MMA e, por fim, o iniciador 2,2' azobis-(2-isobutironitrila), AIBN (Vazo® 64, DuPont), na razão molar de 200 MMA:1 AIBN. Fez-se uso da fonte Q700 da QSONICA e sonda de ultrassom (diâ. 6,4 mm) com amplitude máxima de $170 \mu \mathrm{m}$, na síntese usou-se amplitude relativa de $26 \%$. O tempo de sonicação ativa foi de $25 \mathrm{~min}$, pulsos de $1 \mathrm{~s}$, (50 min total); após o que as suspensões permaneceram em estufa a $60^{\circ} \mathrm{C}$ por $24 \mathrm{~h}$.

A partir das sínteses foram obtidos filmes finos por duas técnicas diferentes: casting e blade coating. Na técnica de casting, a suspensão de PMMA/NTCPM/ $\mathrm{CHCl}_{3}$ foi depositada em substrato de alumínio à temperatura ambiente, permanecendo em capela para evaporação do solvente por $24 \mathrm{~h}$. Na técnica de blade coating, foi utilizado equipamento desenvolvido no CTI Renato Archer. A deposição dos filmes se deu por arraste da suspensão em substrato de vidro $\left(25 \times 25 \mathrm{~mm}^{2}\right)$, regulando-se a espessura da camada úmida com ajuste do espaçamento da lâmina (blade) por micrômetro. A caracterização elétrica dos filmes foi feita no Centro de Componentes Semicondutores na Unicamp. Mediu-se a resistência de folha $\left(\rho_{\mathrm{s}}\right)$ pelo método de van der Pauw para quatro pontas, utilizando-se o instrumento Agilent B2912A. As Equações 1 e 2 fornecem a resistência de folha $\left(\rho_{\mathrm{s}}\right)$ e a condutividade elétrica $(\sigma)$, respectivamente. Em que $R 1$ e $R 2$ são as resistências medidas em direções ortogonais do corpo de prova, $f$ o fator de correção devido à anisotropia e $w$ a espessura do filme.

$$
\begin{aligned}
& \rho_{s}=\frac{\pi}{\ln 2} \frac{R 1+R 2}{2} f\left(\frac{R 1}{R 2}\right) \\
& \sigma=\frac{1}{\rho_{s} w}
\end{aligned}
$$

\section{RESULTADOS E DISCUSSÃO}

Os filmes de nanocompósitos de PMMA/NTCPM produzidos por casting (1\% NTCPM e $26 \%$ amplitude do ultrassom) apresentaram propriedades elétricas típicas de materiais 


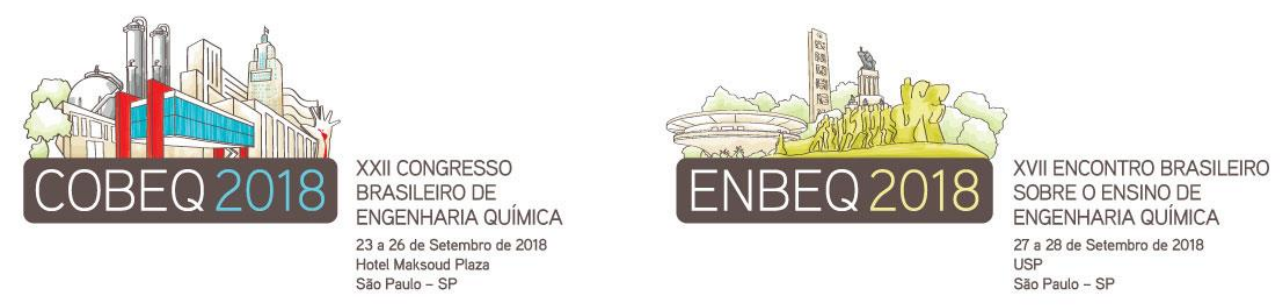

semicondutores (Tabela 1). Os filmes foram produzidos sobre substrato de folha de alumínio, facilmente destacáveis, e apresentavam uma superfície opaca no lado exposto ao ambiente e uma superfície brilhante em contato com o alumínio. As condutividades elétricas das duas superfícies do filme mostraram algumas diferenças, com valores maiores para o lado opaco. Isto pode ser devido, em parte, a um melhor contato das pontas dos eletrodos de medida na superfície opaca; e, em parte, aos grupos funcionais do PMMA que se rearranjam nas superfícies. A formação de distintas rugosidades na superfície dos filmes, não avaliadas aqui, se deve ao processo de evaporação do solvente que tem efeitos distintos nos dois lados do filme. O PMMA contém grupos hidrofóbicos (metileno, $\alpha$-metil e ester-metil) e hidrofílicos (carbonila) em cada unidade. As macromoléculas tendem a minimizar a energia livre de superfície por rearranjo. No PMMA três tipos de movimentos podem ser considerados: segmental, macromolecular e o rotacional dos grupos laterais. Na formação dos filmes por

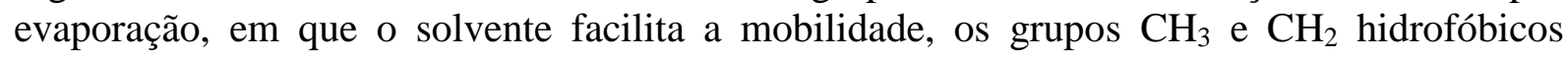
seriam expostos ao ar e os grupos $\mathrm{C}=\mathrm{O}$ para o interior do filme (Kaczmarek et al., 2006).

Tabela 1 - Caracterização elétrica dos filmes de PMMA/NTCPM obtidos via casting

\begin{tabular}{ccccc}
\hline Amostra & Superfície & $\rho_{\mathrm{s}}(\mathrm{k} \Omega / \mathrm{sq})$ & $\mathrm{w}(\mu \mathrm{m})$ & $\bar{\sigma}(\mathrm{S} / \mathrm{m})$ \\
\hline \multirow{2}{*}{ Casting } & Brilhante & 35,60 & 267 & 0,11 \\
& Opaco & 3,82 & 26,98 \\
\hline
\end{tabular}

Tabela 2 - Caracterização elétrica dos filmes PMMA/NTCPM obtidos via blade coating

\begin{tabular}{cccc}
\hline Amostra & Teor NTCPM (\%) & Amplitude US (\%) & $\rho \mathrm{s}(\mathrm{k} \Omega / \mathrm{sq})$ \\
\hline$\# 15$ & $1 \%$ & $26 \%$ & 135,8 \\
$\# 22$ & $1 \%$ & $26 \%$ & 466,5 \\
$\# 73$ & $2 \%$ & $50 \%$ & 93,4 \\
$\# 75$ & $2 \%$ & $50 \%$ & $6,510^{\circ}$ \\
$\# 81$ & $2,2 \%$ & $38 \%$ & $6,310^{\circ}$ \\
$\# 89$ & $2,2 \%$ & $38 \%$ & 74,2 \\
$\# 85$ & $2 \%$ & $26 \%$ & $6,010^{\circ}$ \\
$\# 88$ & $2 \%$ & $26 \%$ & 66,8 \\
\hline
\end{tabular}

Além dos filmes de PMMA/NTCPM produzidos por casting a caracterização elétrica foi realizada com filmes produzidos pela técnica de blade coating (Tabela 2), com diferentes espessuras. As amostras por blade coating \#15 e \#22, esta última de menor espessura, são de nanocompósitos com 1\% de NTCPM e 26\% de Amplitude, mesmas condições das amostras por casting (Tabela 1). Ao reduzir-se a espessura dos filmes, observa-se um aumento de cerca uma ordem de grandeza na resistência de folha, amostra \#22 semitransparente, em relação às amostras \#15 e por casting, ambas negras. Foram, também, preparados filmes por blade coating, amostras \#73, \#75, \#81, \#85, \#88 e \#89 (Tabela 2), com os nanocompósitos de PMMA/NTCPM sintetizados em outro trabalho do grupo (Bressanin, 2017), em que o teor de nanotubos e a amplitude da sonda variaram. Estes nanocompósitos haviam apresentado boas propriedades elétricas quando preparados por casting: 3,5 e $0,6 \mathrm{k} \Omega / \mathrm{sq}$ ou 2,5 e $10,4 \mathrm{~S} / \mathrm{m}$; w = 111 e $166 \mu \mathrm{m}$, com 1\% e 2,2\% NTCPM e 26\% e 38\% Amplitude, respectivamente. Ressaltase que para uma mesma condição de síntese foi produzido mais de um filme, pois o equipamento utilizado no blade coating permite a regulagem da espessura.

Na Figura 1 são mostradas as imagens das amostras \#73, típica dos filmes considerados semitransparentes, e \#85 dos filmes transparentes, isto é, de maior e menor espessura, respectivamente. Observa-se (Tabela 2) que os filmes semitransparentes (\#73, \#89 e \#88) 


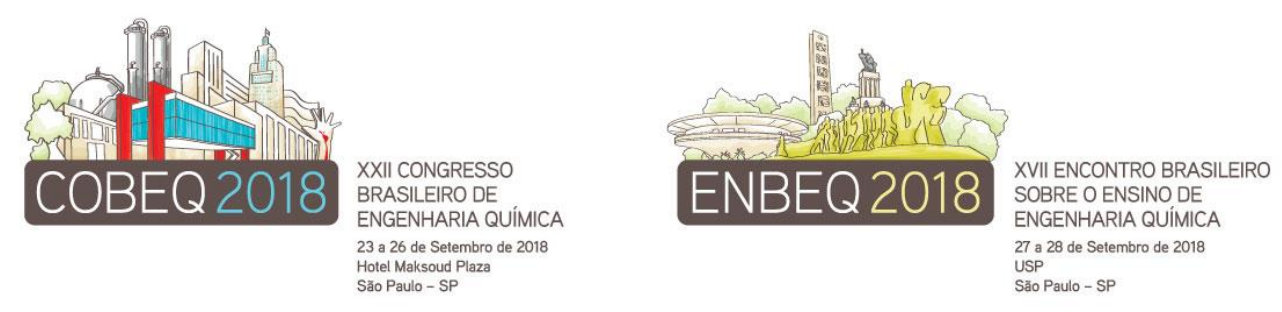

apresentaram resistência de folha cerca de seis ordens inferiores aos filmes semitransparentes (\#75, \#81, \#85), produzidos nas mesmas condições de síntese. Filmes finos poliméricos condutores resultam em elevadas resistividades de superfície. Ainda assim, são valores comuns de materiais semicondutores para distintas aplicações, como filmes antiestáticos semitransparentes ou semicondutores para eletrodomésticos (Ma et al, 2010).

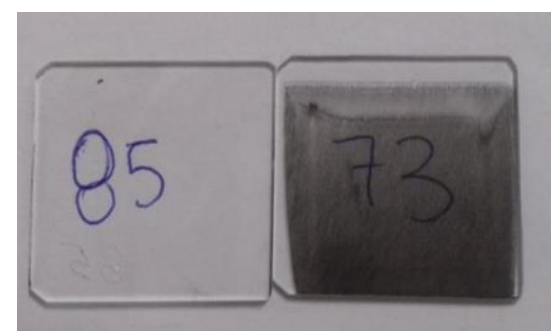

Figura 1 - Foto das amostras \#85 e \#73 produzidas por blade coating.

\section{CONCLUSÕES}

A condutividade elétrica do filme de nanocompósito de PMMA/NTCPM obtido via casting $\sigma \sim 1 \mathrm{~S} / \mathrm{m}$ é típica de material condutor, indicando que a polimerização in situ associada ao ultrassom foi eficiente em dispersar os NTCPM na matriz polimérica. A técnica de blade coating para preparação de filmes finos tem potencial para obter filmes semicondutores poliméricos semitransparentes com resistência de folha de cerca $60 \mathrm{k} \Omega / \mathrm{sq}$. $\mathrm{O}$ compromisso entre transparência e resistência de folha é muito importante para obter filmes finos poliméricos como eletrodos transparentes para aplicações em displays.

Agradecimentos: M.Sc. Marina FC de Andrade (FEQ/Unicamp), CCS/Unicamp, CTI Renato Archer, Depto. de Física da UFMG, Fapesp, Capes e CNPq.

\section{REFERÊNCIAS}

BRESSANIN, JM Síntese via polimerização in situ e caracterização de nanocompósitos condutores elétricos de PMMA em função do teor de nanotubos de carbono e da energia ultrassônica. FEQ, Unicamp. 2017. Dissertação.

GROSSIORD, N; LOOS, J; REVEG, O; KONING, CE Toolbox for dispersing carbon nanotubes into polymers to get conductive nanocomposites.Chem.Mater. 18, 5, 1089-99, 2006

IIJIMA, S. Helical microtubules of graphitic carbon. Nature, v. 354, n. 56, 1991.

KACZMAREK H, CHABERSKA, H; The influence of UV-irradiation and support type on surface properties of poly(methyl methacrylate) thin films, Appl. Surf. Sci. 252, 8185-92, 2006.

MA, P-C; SIDDIQUI, NA; MAROM G; KIM, J-K Dispersion and functionalization of carbon nanotubes for polymer-based nanocomposites: A review. Compos. Part A, 41, 1345-67, 2010.

MITTAL, G; DHAND, V; RHEE, YK; PARK, S-J; LEE, WR; A review on carbon nanotubes and graphene as fillers in reinforced polymer nanocomposites;J.Ind.Eng.Chem.21,11-25,2015

SUSLICK, KS; PRICE, GJ Applications of ultrasound to materials chemistry. Annual Review of Mat. Sci. v. 29, p. 295-326, 1999. 